\title{
Natural Convection in a Porous Cavity Filled with Nanofluid in the Presence of Isothermal Corrugated Source
}

\author{
Islam Bouafia ${ }^{1}$, Razli Mehdaoui ${ }^{2}$, Syham Kadri ${ }^{2,3^{*}}$, Mohammed Elmir ${ }^{1,2}$ \\ ${ }^{1}$ Mechanical Modeling and Experimentation Laboratory, Tahri Mohamed University, Bechar 08000, Algeria \\ ${ }^{2}$ Energarid Laboratory, Tahri Mohamed University, Bechar 08000, Algeria \\ ${ }^{3}$ Laboratory of Semiconductor Devices Physics, Tahri Mohamed University, Bechar 08000, Algeria
}

Corresponding Author Email: kadri.syham@univ-bechar.dz

https://doi.org/10.18280/ijht.380208

Received: 27 January 2020

Accepted: 14 May 2020

\section{Keywords:}

natural convection, nanofluid, porous medium, corrugated source, finite elements method

\begin{abstract}
The present paper is focused on the study of natural convection in a porous square cavity saturated by a nanofluid $\left(\mathrm{Al}_{2} \mathrm{O}_{3}\right.$-Water) in the presence of a corrugated heat source. The horizontal walls and the hatched parts of the vertical walls are considered adiabatic. The corrugated portions of the vertical walls are maintained at uniform and constant temperatures. The basic equations describing the natural convection flow consist of mass conservation, Darcy-Brinkman and energy were solved by means of finite element method. For the physical parameters of $\left(\mathrm{Al}_{2} \mathrm{O}_{3}\right.$-water) nanofluid, we use the Brinkman and Maxwell models. The results are presented as isotherms, streamlines, average Nusselt number, velocity and temperature profiles for various combinations of Modified Rayleigh number, volume fraction of nanoparticles, amplitude and both sources position. It is found that the heat transfer increases with the increase of Modified Rayleigh number, volume fraction and amplitude. The hot source is at the bottom and the cold source is at the top is the optimal position of the two sources to evacuate better the convection heat transfer.
\end{abstract}

\section{INTRODUCTION}

Natural convection in porous media saturated by a fluid is widely studied in the literature. Among these works we include Ingham et al. [1-4]. They studied numerically the fluid displacement using the Darcy-Forchheimer model. For Da $\leq 10^{-5}$, they showed that conduction dominates the heat transfer regardless of the Rayleigh number value. Beyond $\mathrm{Da} \geq 10^{-4}$ the convection is attached to the thermal Rayleigh number evolution. The boundary layer phenomena in rectangular enclosures that contain a porous medium is numerically treated by Tong and Subramanian [5], Lauriat et al. [6] applying the Darcy-Brinkman model [7]. Mehdaoui et al. [8] have developed a comparative study, in the case of free convection, between the Darcy-Brinkman model and the modified Navier-Stokes equations. From the results obtained, they found that the relative error between the two models does not exceed $6 \%$ except for $\mathrm{Ra}=1.5 \times 10^{8}$ (the limit of laminar flow). They concluded that the use of the Darcy-Brinkman model is preferable for laminar flows. Since energy devices sustain a technological progression, they require a good cooling. The use of fluids is inadequate, this is due to the value of the thermal conductivity of fluids by comparing with solids. For a long time, researchers in the field of heat transfer, have tried to find new solutions to optimize the performance of energy devices through the improvement of heat transfer. In 1995, Choi and Eastman [9] carried out a study on the dispersion of solid nanoparticles in a base liquid to improve their thermo-physical properties. This suspension is named nanofluid. Khanafer et al. [10] have conducted studies in this area, they have shown that the variation of heat transfer is more important for nanofluids compared to pure fluids. Among the advantages of nanofluids we can adduce: a large specific surface area compared with conventional solid-liquid suspensions, reduced fouling of nanoparticles for equivalent heat transfer and lower pumping power compared to other suspensions, therefore a lower expenditure.

The phenomenon of natural convection in porous media saturated by a nanofluid has a various application in engineering as, the cooling of electronic components, storage of different types of energy, heat exchangers and thermal buildings. Many researchers have invested in this field, Khanafer et al. have studied the influence of natural convection heat transfer in a confined porous medium saturated by a nanofluid $\left(\mathrm{Al}_{2} \mathrm{O}_{3}\right.$-water $)$ [11, 12]. The simulation of the partial differential equations system is solved with the finite volume method. They showed the influence of some parameters on the heat transfer such as the volume fraction of the nanoparticles, the Darcy and the Rayleigh numbers. The propagation of heat in a confined porous medium saturated by a nanofluid has simulated by Sheikholeslami et al. [13, 14]. They have showed that the Nusselt number increases as the Rayleigh number and the volume fraction increases. On the other hand, it decreases with the increase in the Hartmann number. Revnic et al. [15] have considered the effect of the Brownian motion proposed by Buongiorno [16]. Many theoretical and experimental works have developed in the field of porous medium saturated by a nanofluid, we can cite Baïri et al. [17-20].

The problem of natural convection in a wavy enclosure filled by a porous medium saturated by a nanofluid has exploited by many researchers. Shenoy et al. have recently simulated the effect of corrugated source in a porous cavity saturated by a nanofluid with the finite element method. The 
results obtained enable to deduce that increasing of corrugations numbers decreases the heat transfer [21, 22]. Also, amplification of the amplitude of the concave wall improves convective heat transfer. Near convex walls, the phenomenon reverses. Sheremet et al. have presented a numerical analysis of natural convection in a partially heated corrugated porous cavity saturated by a nanofluid in the presence of a magnetic field [23, 24].

This paper studies numerically the phenomenon of natural convection in a porous cavity saturated by a nanofluid $\left(\mathrm{Al}_{2} \mathrm{O}_{3}-\right.$ Water) with partially corrugated vertical walls. Several effects are taken into consideration such as: the modified Rayleigh number, the volume fraction of the nanoparticles, the amplitude and the position of corrugated sources. The objective of our work is to search the optimal amplitude and position of the source in order to find the favorable cooling conditions of the electronic components and storage different types of energy.

\section{GEOMETRY}

AND

MATHEMATICAL FORMULATION

The physical model is shown in Figure 1. It consists of a stationary two-dimensional problem of natural convection in a porous enclosure of dimension $(\mathrm{LxH})$ saturated with a nanofluid $\left(\mathrm{Al}_{2} \mathrm{O}_{3}\right.$-Water). The hatched part of the vertical walls and the two horizontals walls are considered adiabatic. The unhatched part of the vertical walls is partially corrugated and maintained at constant temperatures. This corrugation is traced by the second-order Bézier curve.

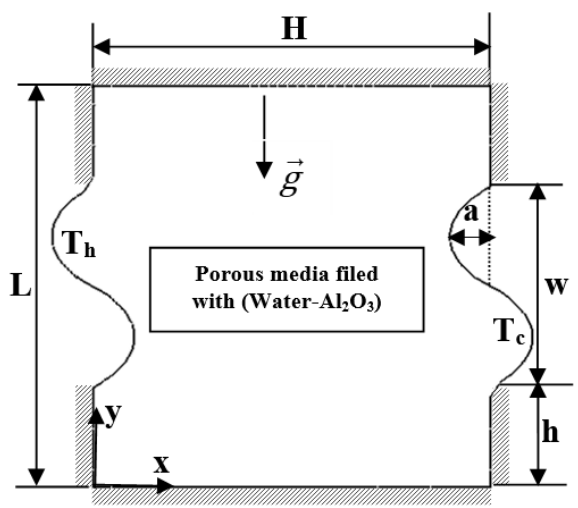

Figure 1. Physical model

The nanofluid is assumed incompressible and the flow is laminar. The porous medium and the nanofluid are homogeneous and isotropic substances. Thermo-physical properties of nanofluid are supposed constant. Also, the base fluid and the nanoparticles are in thermal equilibrium and they flow at the same velocity. The Boussinesq approximations are undertaken to be valid. In the present study, the radiation effects, Brownian movement of nanoparticles and chemical reactions during flow are neglected. Another important hypothesis assumes that the matrix of the porous medium is in deformable and in thermal equilibrium with the nanofluid and that its diffusion is neglected.

The dimensional system of equations is written as:

$$
\frac{\partial u}{\partial x}+\frac{\partial v}{\partial y}=0
$$

$$
\begin{gathered}
\frac{\mu_{n f}}{k^{*}} u+\frac{\partial P}{\partial x}=\mu_{n f}\left(\frac{\partial^{2} u}{\partial x^{2}}+\frac{\partial^{2} u}{\partial y^{2}}\right) \\
\frac{\mu_{n f}}{k^{*}} v+\frac{\partial P}{\partial y}=\mu_{n f}\left(\frac{\partial^{2} v}{\partial x^{2}}+\frac{\partial^{2} v}{\partial y^{2}}\right)-\rho_{n f} g \\
u \frac{\partial T}{\partial x}+v \frac{\partial T}{\partial y}=\frac{k_{m}}{\rho c_{p}}\left(\frac{\partial^{2} T}{\partial x^{2}}+\frac{\partial^{2} T}{\partial y^{2}}\right)
\end{gathered}
$$

Based on the suspension theory, the effective density, the heat capacity and the thermal expansion coefficient of a nanofluid are defined by the following relationships:

$$
\rho_{n f}=1-\phi \rho_{f}+\phi \rho_{s}
$$

$$
\begin{gathered}
\rho c_{p_{n f}}=1-\phi \quad \rho c_{p_{f}}+\rho c_{p}{ }_{s} \\
\beta_{n f}=1-\phi \beta_{f}+\phi \beta_{s}
\end{gathered}
$$

The dynamic viscosity and the thermal conductivity are calculated respectively from the Brinkman and Maxwell models:

$$
\begin{gathered}
\mu_{n f}=\frac{\mu_{f}}{1-\phi}{ }^{2.5} \\
\frac{k_{n f}}{k_{f}}=\frac{k_{s}+2 k_{f}+2 k_{s}-k_{f} \phi}{k_{s}+2 k_{f}-k_{s}-k_{f} \phi}
\end{gathered}
$$

The thermal diffusivity of the nanofluid is given by:

$$
\alpha_{n f}=\frac{k_{n f}}{\rho c_{p}{ }_{n f}}
$$

Based upon the previous assumptions and introducing the following dimensionless variables:

$$
\begin{gathered}
\left(x^{*}, y^{*}\right)=\frac{(x, y)}{H},\left(u^{*}, v^{*}\right)=\frac{(u, v) H}{\alpha_{f}}, \\
p^{*}=\frac{p H^{2}}{\rho_{f} \alpha_{f}^{2}}, T^{*}=\frac{T-T_{h}}{T_{c}-T_{h}},
\end{gathered}
$$

After replacing these last variables (11) in the Eqns. (1-4), the dimensionless forms of the governing equations under steady state condition are expressed as following: 


$$
\begin{gathered}
\frac{\partial u^{*}}{\partial x^{*}}+\frac{\partial v^{*}}{\partial y^{*}}=0 \\
\frac{1}{(1-\phi)^{2.5}} \frac{\operatorname{Pr}}{D a} u^{*}+\frac{\partial p^{*}}{\partial x^{*}} \\
=\frac{1}{(1-\phi)^{2.5}} \operatorname{Pr}\left(\frac{\partial^{2} u^{*}}{\partial x^{* 2}}+\frac{\partial^{2} u^{*}}{\partial y^{* 2}}\right) \\
\frac{1}{(1-\phi)^{2.5}} \frac{\operatorname{Pr}}{\operatorname{Da} v^{*}+\frac{\partial p^{*}}{\partial y^{*}}} \\
=\frac{1}{(1-\phi)^{2.5}} \operatorname{Pr}\left(\frac{\partial^{2} v^{*}}{\partial x^{* 2}}+\frac{\partial^{2} v^{*}}{\partial y^{* 2}}\right) \\
+\frac{R a^{*}}{D a} \operatorname{Pr}\left[(1-\phi)+\phi \frac{\rho_{s} \beta_{s}}{\varphi_{f} \beta_{f}}\right] T^{*} \\
u^{*} \frac{\partial T^{*}}{\partial x^{*}}+v^{*} \frac{\partial T^{*}}{\partial y^{*}}=R R_{k} R_{\alpha}\left(\frac{\partial^{2} T^{*}}{\partial x^{* 2}}+\frac{\partial^{2} T^{*}}{\partial y^{* 2}}\right)
\end{gathered}
$$

The Rayleigh number, Darcy number, modified Rayleigh number, Prandtl number, conductivity and thermal diffusivity ratios are defined, respectively, as:

$$
\begin{gathered}
R a=\frac{\rho_{f} \beta_{f} H^{3} g \Delta T}{\mu_{f} \alpha_{f}} \quad, \quad D a=\frac{k^{*}}{H^{2}} \\
R a^{*}=R a \cdot D a, \quad \operatorname{Pr}=\frac{\mu_{f}}{\rho_{f} \alpha_{f}^{2}} \\
R_{k}=\frac{k_{m}}{k_{n f}}, \quad R_{\alpha}=\frac{\alpha_{n f}}{\alpha_{f}}
\end{gathered}
$$

Figure 2 shows the dimensionless forms of the boundary conditions for the present problem.

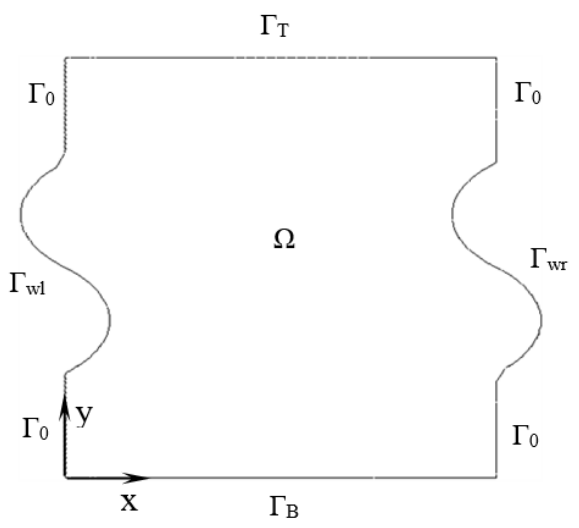

Figure 2. Dimensionless boundaries conditions

We note here that the previous equations are solved in the field $\Omega$ of the configuration Figure 2; the boundary of this field noted $\partial \Omega=\Gamma_{T} \cup \Gamma_{B} \cup \Gamma_{W l} \cup \Gamma_{W r} \cup \Gamma_{0}$ such as $\Gamma_{T}$ and $\Gamma_{B}$

are respectively the Top and Bottom boundary, $\Gamma_{W l}$ and $\Gamma_{W r}$ are respectively the left and right wavy boundaries, $\Gamma_{0}$ are the remaining borders of the left and right walls as illustrated in Figure 2. Table 1 groups the dimensionless boundary conditions.

Table 1. The dimensionless forms of the boundary conditions

\begin{tabular}{ccc}
\hline $\begin{array}{c}\text { Boundaries } \\
\text { conditions }\end{array}$ & $\begin{array}{c}\text { Thermal } \\
\text { boundaries }\end{array}$ & $\begin{array}{c}\text { Hydrodynamic } \\
\text { boundaries }\end{array}$ \\
\hline$\Gamma_{\mathrm{T}}$ & $\frac{\partial T^{*}}{\partial y^{*}}=0$ & $u^{*}=v^{*}=0$ \\
$\Gamma_{\mathrm{B}}$ & $\frac{\partial T^{*}}{\partial y^{*}}=0$ & $u^{*}=v^{*}=0$ \\
& $\frac{\partial T^{*}}{\partial y^{*}}=0$ & $u^{*}=v^{*}=0$ \\
$\Gamma_{0}$ & 0 & $u^{*}=v^{*}=0$ \\
$\Gamma_{\mathrm{wr}}$ & 1 & $u^{*}=v^{*}=0$ \\
$\Gamma_{\mathrm{wl}}$ & & \\
\hline
\end{tabular}

The local and average Nusselt numbers are calculated at the wavy walls from the following relations:

$$
\begin{gathered}
N u_{L}=-\left.\frac{k_{n f}}{k_{f}} \frac{\partial T *}{\partial n}\right|_{\text {Wall }} \\
N u_{a v r}=\frac{1}{l_{w}} \int_{h^{*}}^{h^{*}+\varepsilon} N u_{L} \cdot d n
\end{gathered}
$$

where,

$\mathrm{n}$ is the normal direction of the wall.

$l_{\mathrm{w}}$ is the length of wavy surface.

\section{NUMERICAL METHOD}

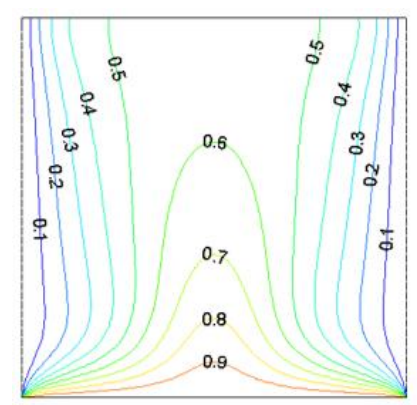

(a)

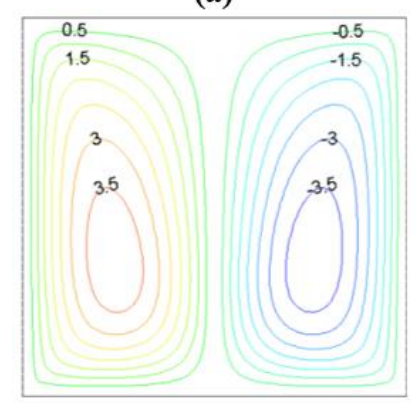

Figure 3. Isotherms and Streamlines for $R a=10^{6}, D a=10^{-4}$, $\operatorname{Pr}=0.71$ and $T^{*}(x, 0)=1$ : comparison present results (a) with the result of Basak el al. [4] (b).

To solve numerically the differential Eqns. (12)-(15) with the boundary conditions, we used the discretization of the 
Galerkin finite element method. The two-dimensional spatial domain is divided into triangular elements (non-orthogonal mesh) and a Lagrange-quadratic interpolation has been chosen. A nonlinear solver has been used and the nonlinear tolerance has been set to $10^{-6}$.

In order to validate our results, we have used as reference some numerical results available in the literature. The first validation consists in studying the phenomenon of natural convection in a porous square cavity saturated by a fluid elaborated by Basak et al. [4]. The results are presented in the form of isotherms, streamlines (Figure 3) for $R a=10^{6}, P r=0.71$ and local Nusselt number variation for $R a=10^{6}, P r=10$ (Figure 4). The Figures show an acceptable concordance between our numerical results and that of Basak.

The second validation is a comparison between our results and that of Shehzad and Nazari [13] in terms of average Nusselt number. During this validation we studied the phenomenon of free convection in a porous cavity saturated by a nanofluid $\left(\mathrm{Al}_{2} \mathrm{O}_{3}\right.$-Water $)$ the results in Table 2 show a concordance between our numerical results and the literature.

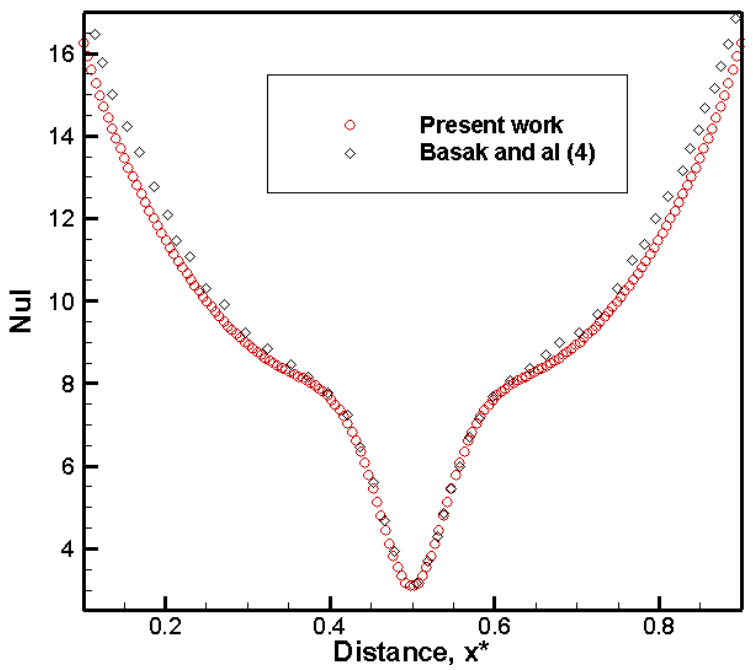

Figure 4. Validation of local Nusselt number with distance $x^{*}$ at the bottom wall for $R a=10^{6}, D a=10^{-3}, P r=10$

Table 2. Validation of average Nusselt number with different Rayleigh number for $D a=10^{-3}, P r=5.83$ and $\varphi=10 \%$

\begin{tabular}{cccc}
\hline \multicolumn{4}{c}{ Average Nusselt number } \\
\hline & Present work & Shehzad $[13]$ & The difference \% \\
\hline $\mathrm{Ra}=10^{5}$ & 2.25 & 2.34 & $3.8 \%$ \\
$\mathrm{Ra}=10^{6}$ & 7.26 & 7.70 & $5.7 \%$ \\
\hline
\end{tabular}

Table 3. Variations of the average Nusselt number of the heat source with the non-uniform grids

\begin{tabular}{ccc}
\hline $\begin{array}{c}\text { Number } \\
\text { of } \\
\text { elements }\end{array}$ & $N u_{\text {avr }}$ & $\Delta=\left|\frac{N u_{a v r, i}-N u_{a v r, 5192}}{N u_{a v r, i}}\right| \times 100 \%$ \\
\hline 700 & 10.1233 & $0.69 \%$ \\
1738 & 10.1407 & $0.52 \%$ \\
5192 & 10.1932 & ------ \\
5380 & 10.1949 & $0.017 \%$ \\
5568 & 10.1962 & $0.030 \%$ \\
\hline
\end{tabular}

The mesh test is performed in a porous cavity saturated by a nanofluid with a partially corrugated wall, we set the following parameters at $R a^{*}=10^{4}, \varphi=10 \%, a=0.1$ and $h^{*}=0.25$.
Table 3 presents the mesh effect on the average Nusselt number at the wavy hot source.

According to the test performed in Table 3, the optimal mesh is around 5192 elements, Figure 5 shows the distribution of the optimal mesh chosen in our model.

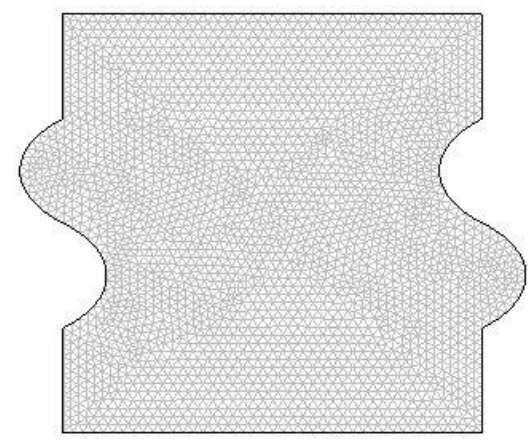

Figure 5. Optimal mesh grid

\section{RESULTS AND DISCUSSION}

To describe the structure of the thermal and hydrodynamic flow of natural convection in a porous cavity saturated by a nanofluid $\left(\mathrm{Al}_{2} \mathrm{O}_{3}\right.$-Water $)$ with partially corrugated wall, the following parameters are fixed: $D a=10^{-2}, \varepsilon=0.5, A=1, n=1$ and $R_{k}=1$ and the Prandtl number of water is $P r=5.83$ [13]. Thermophysical properties of the base fluid and the nanoparticles are presented in Table 4.

Table 4. Thermophysical properties of water and nanoparticles at $\mathrm{T}=25^{\circ} \mathrm{C}[13]$

\begin{tabular}{ccc}
\hline Physical property & Water & Nanoparticles \\
\hline $\mathrm{Cp}[\mathrm{J} / \mathrm{Kg}]$ & 4179 & 765 \\
$\rho\left[\mathrm{Kg} / \mathrm{m}^{3}\right]$ & 997.1 & 3960 \\
$\mathrm{k}[\mathrm{W} / \mathrm{m} \mathrm{k}]$ & 0.613 & 40 \\
$\beta\left[\mathrm{K}^{-1}\right]$ & $21 \times 10^{-5}$ & $0.85 \times 10^{-5}$ \\
$\mu[\mathrm{kg} / \mathrm{m} . \mathrm{s}]$ & $8.55 \times 10^{-4}$ & ------- \\
\hline
\end{tabular}

\subsection{The modified Rayleigh number effect}

Figure 6 shows the variation of isotherms and streamlines for different values of the modified Rayleigh number $R a^{*}$ (10; $10^{2} ; 10^{3}$ and $\left.10^{4}\right)$ and the control parameters: $\phi=2 \%, a^{*}=0.1$, $h^{*}=0.25$. We note that for $R a^{*}=10$. (See Figure 6.a), the isotherms are almost parallel to the gravitational acceleration field $\left(\Delta T^{*} \perp \vec{g}\right)$ thus giving a pseudo-thermal stratification, conduction is the predominant mechanism of heat transfer in this study. Increase the modified Rayleigh number $\left(R a^{*} \geq 10^{2}\right.$, Figures 6.b, 6.c, 6.d), the isotherms deform until the temperature gradient becomes parallel to the acceleration field of gravity $\left(\Delta \mathrm{T}^{*} \| \vec{g}\right)$, the convective exchange dominates the entire cavity which promotes the rate of heat dissipation.

We also note that for different $\mathrm{Ra}^{*}$ values the thickness of the thermal boundary layer close to the corrugated walls decreases, the isotherms close to these walls are condensed and always remains parallel to the differentially heated walls, the conductive exchange is favored. For the distribution of the streamlines we note that for different values of $\mathrm{Ra}^{*}$, the structure of the flow is represented in a single central cell, the intensity of the flow and the force of circulation increases and becomes more significant in the whole cavity with the increase of $R a^{*}$ which favors the convective flow regime compared to 
the conductive regime thus confirming the isothermal results. The vortex keeping the same position in the heart of the cavity but it expands with the growth of $R a^{*}$. Also, when the modified Rayleigh number increases, the thickness of the hydrodynamic boundary layer shrinks which is clear in the important values of the density of the current lines close to the corrugated rigid walls.
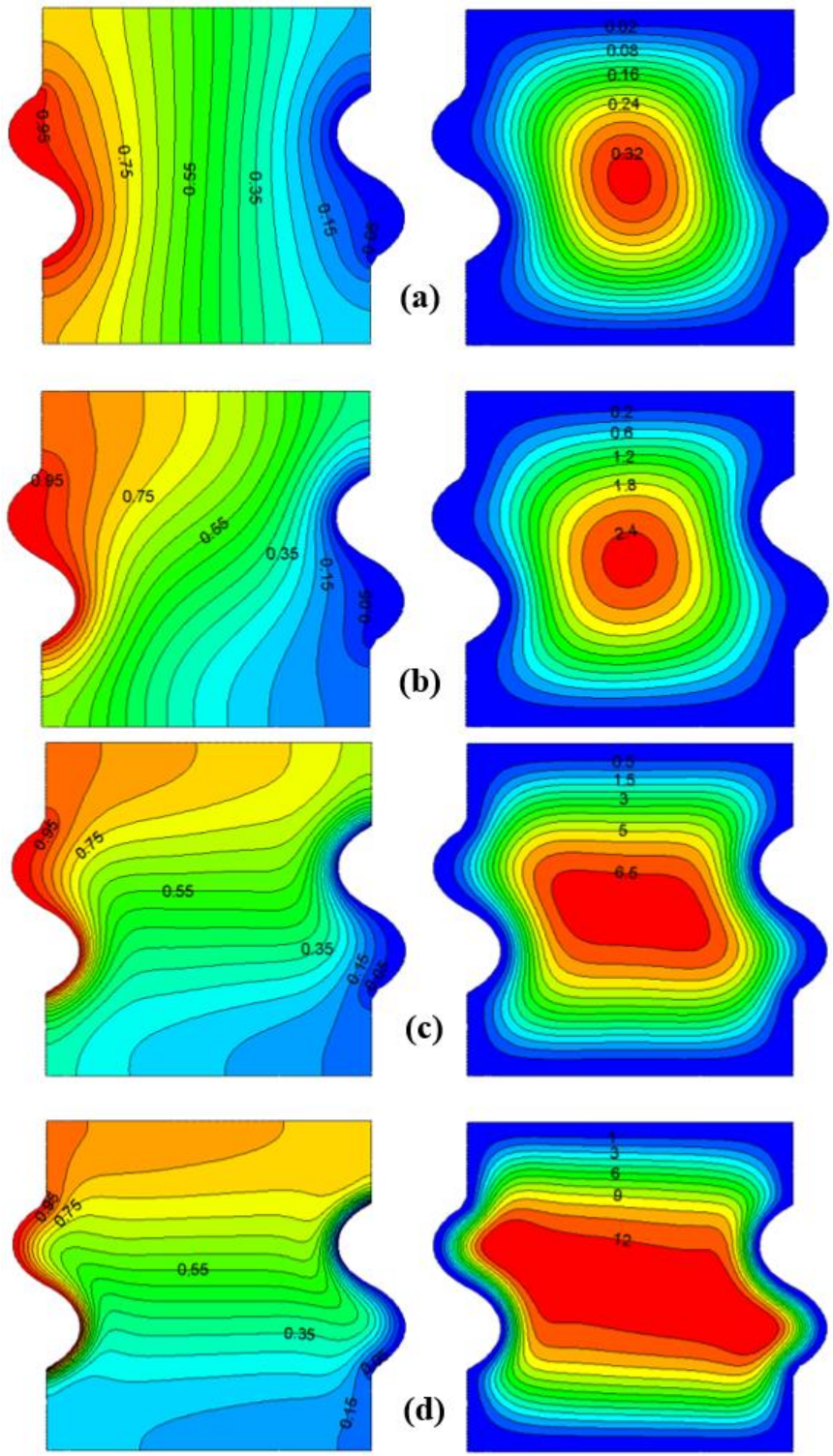

Figure 6. Isotherms and Streamlines at different modified Rayleigh number: (a) $R a^{*}=10$, (b) $R a^{*}=10^{2}$, (c) $R a^{*}=10^{3}$, (d) $R a^{*}=10^{4}$

Figure 7 shows the variation of the stream function along the cavity at $y^{*}=0.5$ for different modified Rayleigh values. We observe that the stream function is almost parabolic. Whatever the value of $R a^{*}$, the flow intensity near the corrugated walls is almost zero $(\psi=0)$ and takes a maximum value at the center of the cavity. Beyond $R a^{*}>10^{2}$; the stream function value is maintained constant over a large length distance of the cavity, which explains the expansion of the central vortex. The evolution of the stream function explains the importance of the influence of buoyancy forces on viscous forces. The convective flow regime encompasses the entire cavity with the increase of $R a^{*}$.

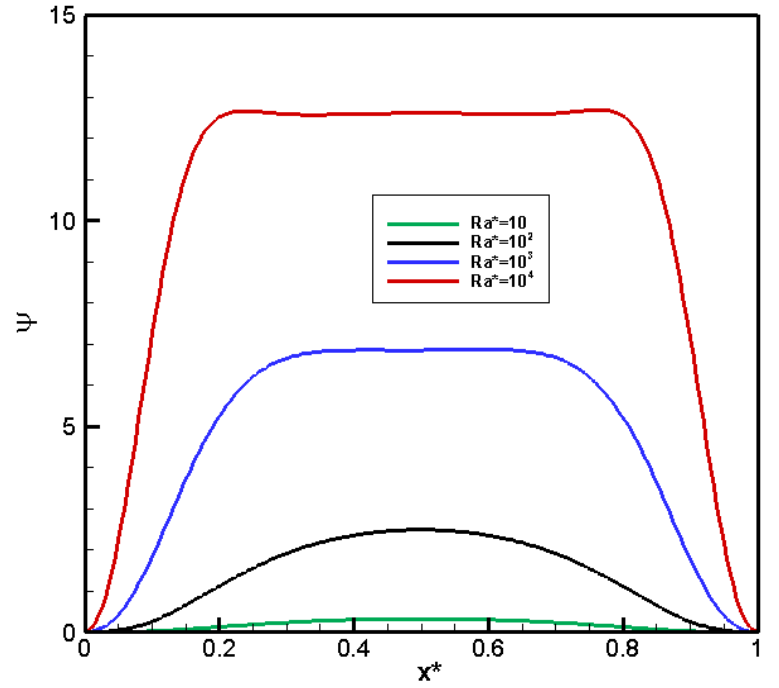

Figure 7. Variation of the stream function at $(0,0.5)$ to $(1$, $0.5)$

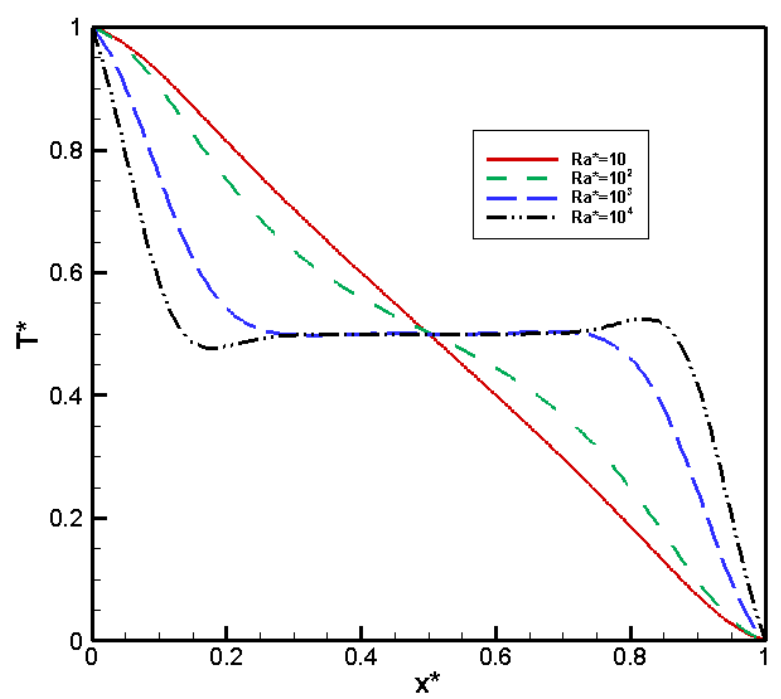

Figure 8. Temperature evolution at $(0,0.5)$ to $(1,0.5)$

Figure 8 illustrates the temperature evolution as a function of the cavity length at $y^{*}=0.5$ for different modified Rayleigh values. The figure shows a critical point located in the center of the nucleus. There is symmetry with respect to this point. At $R a^{*}=10$, the temperature variation is almost linear. As the modified Rayleigh number increases, the evolution of the temperature loses its linearity. Near the hot corrugated wall, the increase in the modified Rayleigh number decreases the temperature value which confirms the isothermal observations. Close to the cold corrugated wall, the phenomenon is reversed.

\subsection{The source amplitude effect}

In this part we have studied the effect of amplitude $a^{*}$. For this we have varied the hot source amplitude between 0.05 0.35 when the cold source amplitude is fixed at $a^{*}=0.10$. The two sources are fixed in the middle of the vertical walls.

Figure 9 represents the evolution of the average Nusselt number as a function of the amplitude for different modified Rayleigh numbers at $\phi=2 \%$. We can notice dependence between the average Nusselt number and the amplitude of the hot corrugated wall whose the amplification of the latter increases the heat transfer rate from the increase in the wall- 
fluid exchange surface. This amplification takes place in a monotonous way. We also observe that increasing the modified Rayleigh number increases the value of the average Nusselt number.

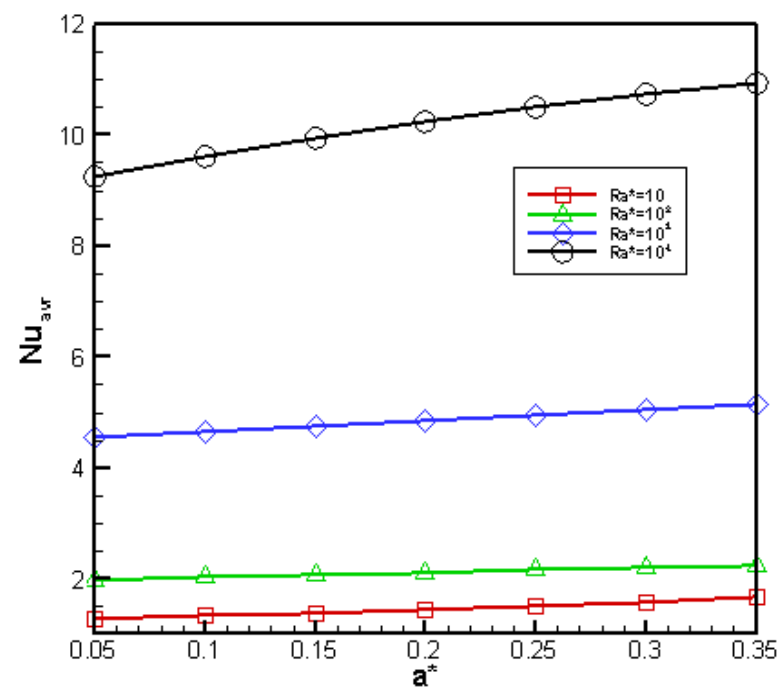

Figure 9. Variation of the average Nusselt number with various amplitude of hot source at different modified

Rayleigh number for $h^{*}=0.25$ and $\phi=2 \%$
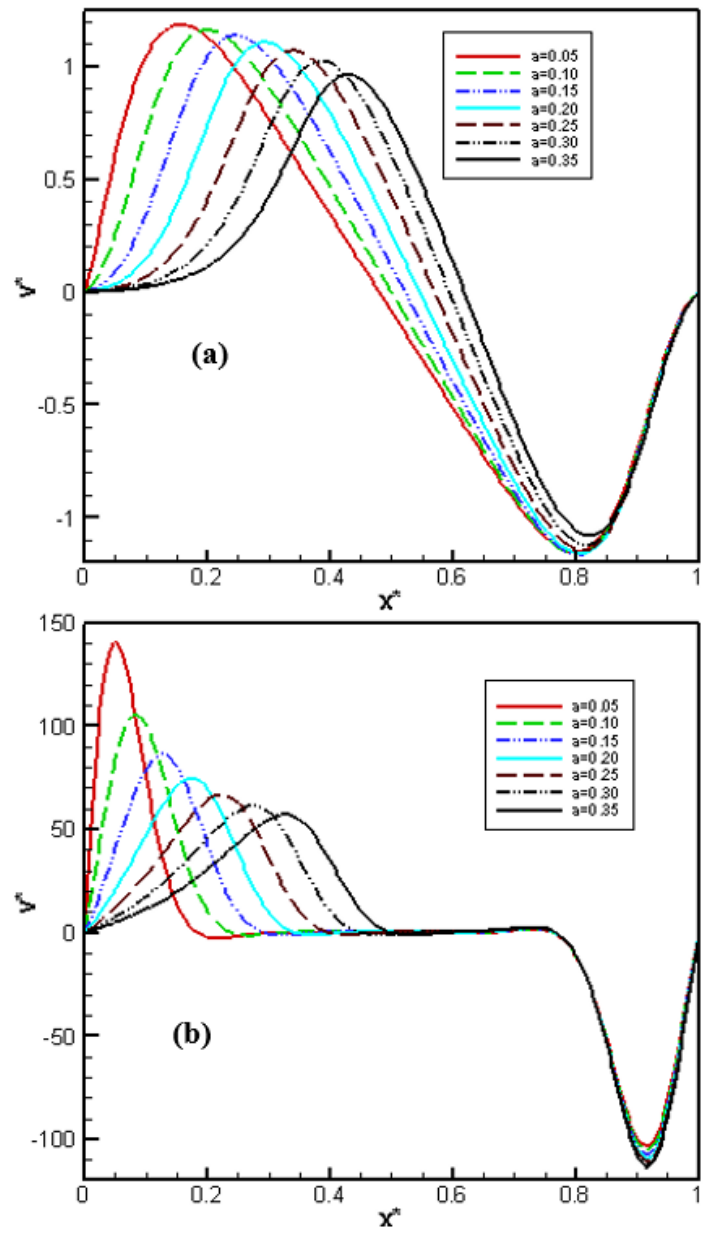

Figure 10. Variation of the vertical component of velocity $\mathrm{v}^{*}$ at $(0,0.5)$ to $(1,0.5)$ for $\mathrm{h}^{*}=0.25$ and $\phi=2 \%$ : (a) $R a^{*}=10$ (b) $R a^{*}=10^{4}$

The Figure 10, shows the variation of the vertical component of velocity $v^{*}$ at $y^{*}=0.5$ along the width of the cavity for two cases (a): $\mathrm{Ra}^{*}=10$ and (b): $R a^{*}=10^{4}$. The first remark is that the velocity increases with the increase of the modified Rayleigh number. Thus, we note a deceleration of the fluid as the amplitude increases especially for $R a^{*}=10^{4}$ or the convective regime is dominant. This means that the effect of adhesion to the wall increases with increasing amplitude because the point of the maximum value approaches the middle of the cavity. Near the fixed cold wall, the velocity remains constant.

\subsection{The nanoparticle volume fraction effect}

To describe the influence of the volume fraction of nanoparticles on heat transfer in a partially wavy cavity, the following control parameters are fixed: $h^{*}=0.25$ and $a^{*}=0.1$.

The variation of $N u_{a v r}$ as a function of the nanoparticles volume fraction is illustrated in Figure 11 for different values of the modified Rayleigh number. We observe that the average Nusselt number (the rate of heat transfer) increases with the increase in the volume fraction of the nanoparticles $\phi$, which means that the thermal conductivity increased, moreover the amplification of the modified Rayleigh number $R a^{*}$ increases the buoyancy force thus the average Nusselt number. On the other hand the hydrodynamic flow of the fluid becomes slower with the increase in the volume fraction of the solid because it is strongly attached to the walls this depends on the increase in the viscosity of the nanofluid (the hydrodynamic flow becomes slower because that it depends on the interactions between the layers of the fluid and the effect of adhesion to the walls) (see Table 5).

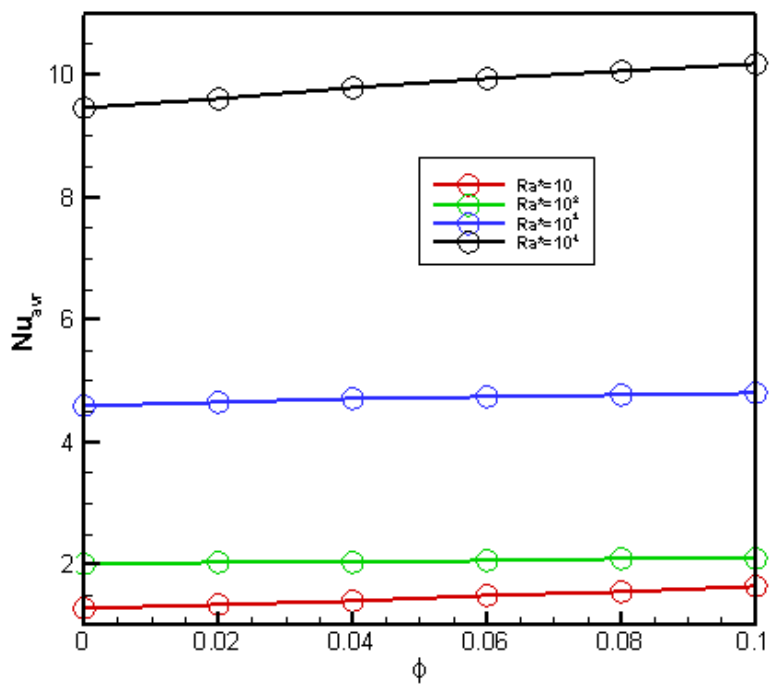

Figure 11. Variation of the average Nusselt number with the volume fraction of the nanoparticles at different modified Rayleigh numbers

Table 5. Maximal velocity as function as Modified Rayleigh number and volume fraction for nanoparticles

\begin{tabular}{|c|c|c|c|c|c|c|}
\hline \multicolumn{7}{|c|}{$|U|_{\max }$} \\
\hline$\phi$ & $\phi=$ & $\phi=$ & $\phi=$ & $\phi=$ & $\phi=$ & $\phi=$ \\
\hline$R a^{*}$ & 0.00 & 0.02 & 0.04 & 0.06 & 0.08 & 0.10 \\
\hline 10 & 1.45 & 1.35 & 1.26 & 1.17 & 1.08 & 1.00 \\
\hline $10^{2}$ & 11.90 & 11.43 & 10.93 & 10.41 & 9.87 & 9.32 \\
\hline $10^{3}$ & 52.34 & 51.76 & 51.04 & 50.24 & 49.31 & 48.27 \\
\hline $10^{4}$ & 171.55 & 170.65 & 169.37 & 168.22 & 166.85 & 165.12 \\
\hline
\end{tabular}




\subsection{The effect of the hot and the cold source position}
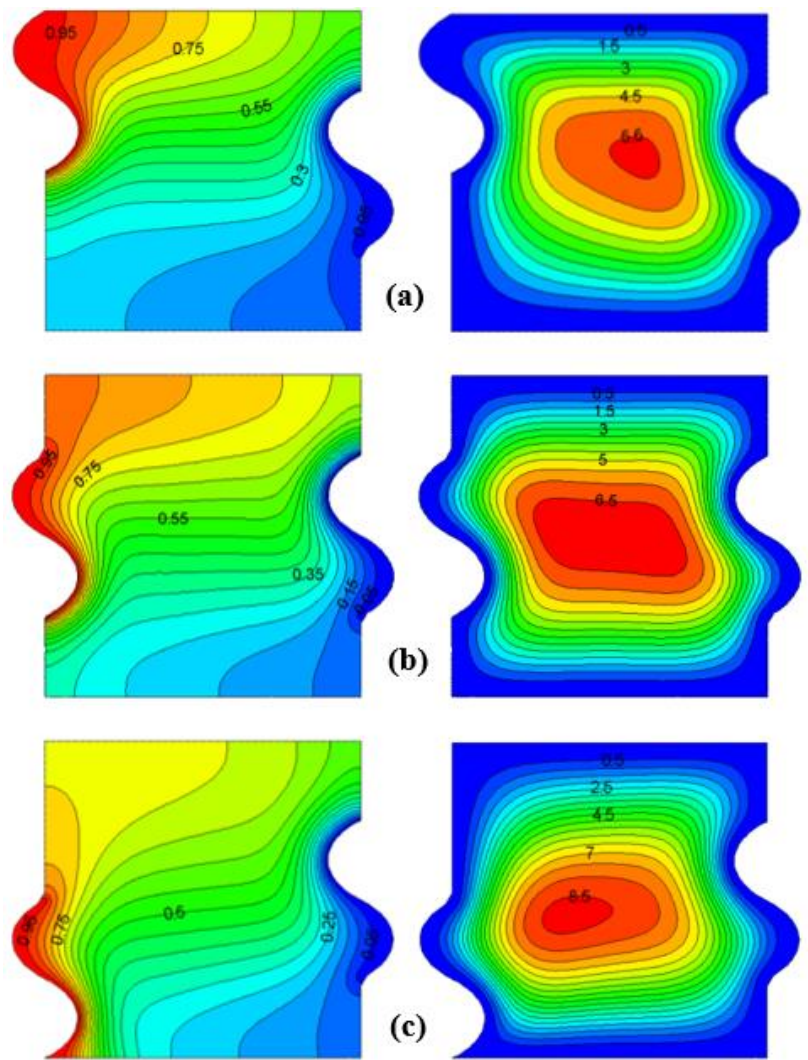

Figure 12. Temperature and Stream function Distribution at different position of hot source: (a) $h^{*}=0.5$, (b) $h^{*}=0.25$, (c) $h^{*}=0$
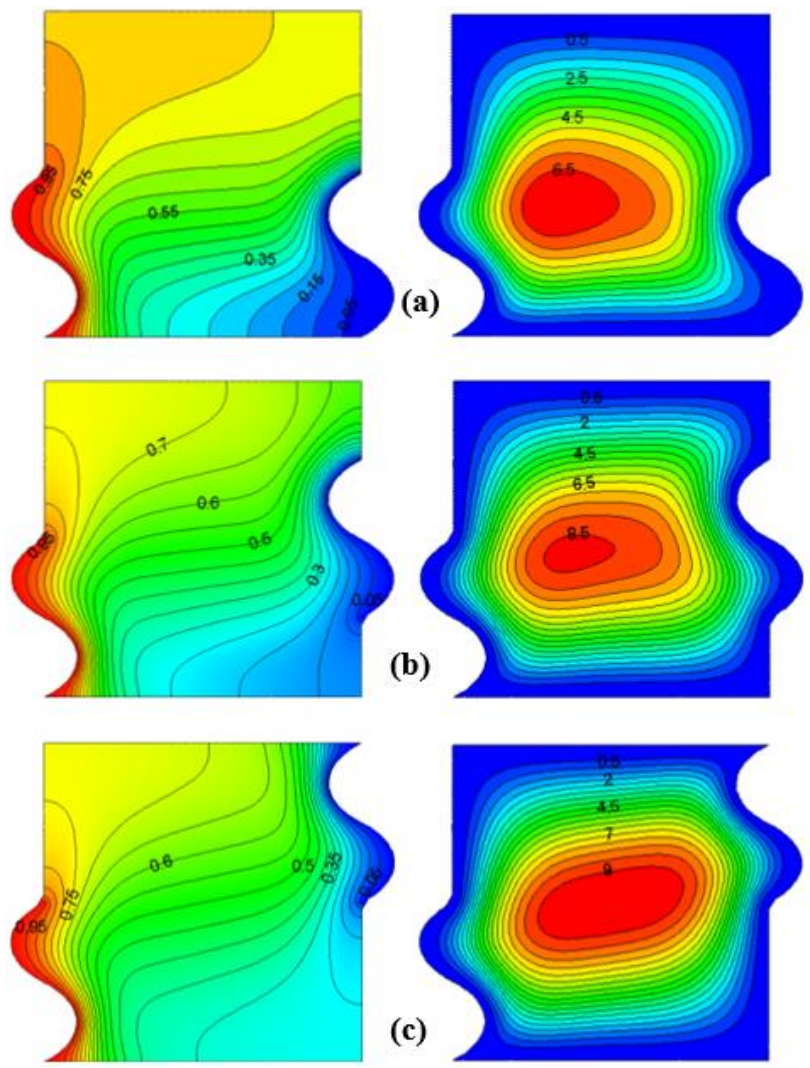

Figure 13. Temperature and Stream function Distribution at different position of cold source: (a) $h^{*}=0$, (b) $h^{*}=0.25$, (c) $h^{*}=0.5$
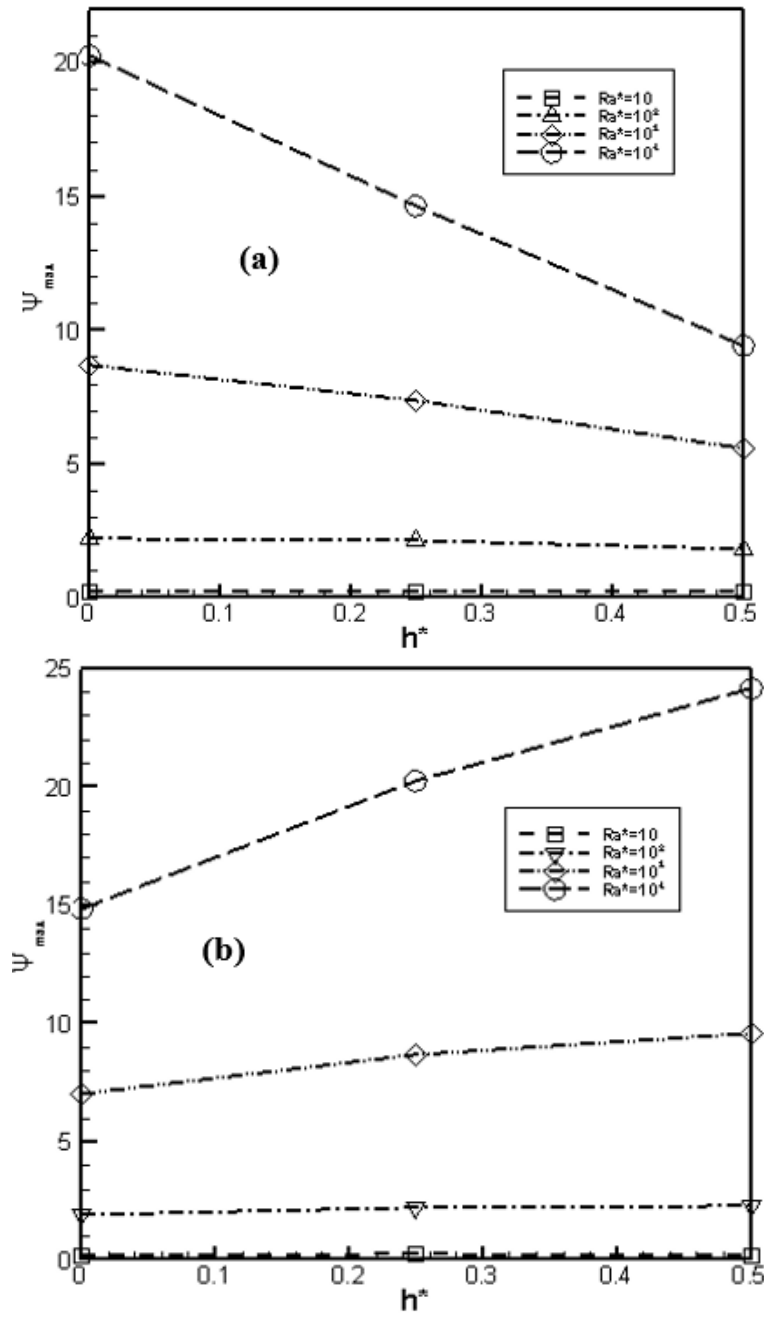

Figure 14. Maximum stream function value of different position; (a) hot source (b) cold source

Figure 12 shows the distribution of isotherms and streamlines for different positions of the corrugated hot source located on the left vertical wall. The cold source always remains fixed at the position $h^{*}=0.25, R a^{*}=10^{3}, a^{*}=0.1$ and $\phi=10 \%$.

It is noted that the isotherms distribution has the shape ensuring a convective transfer mode. There is a zone of the hot fluid near the top part of the hot corrugated wall, this zone shrinks when the hot source takes the bottom position. This means that the bottom position of the hot source ensures good heat dissipation by natural convection. The streamlines distribution is characterized by a single central cell for different positions of the hot source, except that the shape of the vortex changes. The convective flow regime remains dominant. We also observe that the convective flow becomes more and more favorable, when the hot source takes the bottom position of the left vertical wall.

The distribution of isotherms and streamlines for different positions of the corrugated cold source is illustrated in the Figure 13. The following control parameters are fixed: $R a^{*}=10^{3}, \phi=10 \%, a^{*}=0.1$ and the hot source remains at the bottom position $\mathrm{h}^{*}=0$.

From this distribution we notice that the flow force intensity becomes more important as the cold source is moved to the top. This favors the convective regime, so the convective heat transfer regime is gaining momentum, the buoyancy effect is confirmed. See Figure 14. 


\section{CONCLUSION}

In this work, we performed a numerical simulation of natural convection inside a porous enclosure saturated with $\left(\mathrm{Al}_{2} \mathrm{O}_{3}\right.$-water $)$ nanofluid partially wavy. The first results obtained indicate that the increase in the number of modified Rayleigh ensures that the porous medium becomes more permeable, the average Nusselt number and the buoyancy force increase. Consequently, the viscous force improves which translates acceleration in the molecules of the fluid and there after the convective flow is favored. The second results show that the amplitude of the corrugated wall influences the rate of heat transfer in the porous cavity where it enhances the average Nusselt number. Also, the flow velocity of the nanofluid in the enclosure decreases. The third results deduced from the increase in the volume fraction of nanoparticles which implies a decrease in the recirculation rate of nanofluids in the cavity and an improvement in the average Nusselt number. Finally, to ensure heat dissipation, the optimal position of the hot source is presented at the bottom and that of the cold source is at the top, this strongly depends on the density distribution of the hot and cold fluid (density gradient effect).

\section{REFERENCES}

[1] Pop, I., Ingham, D.B. (2001). Convective Heat Transfer: Mathematical and Computational Modelling of Viscous Fluids and Porous Media. 1st Edition, Elsevier, Pergamon.

[2] Nield, D.A., Bejan, A. (2006). Convection in porous media. Springer, Vol. 3. New York.

[3] Vafai, K. (2015). Handbook of Porous Media-CRC Press Taylor \& Francis group. ISBN:978-1-4398-8554-3.

[4] Basak, T., Roy, S., Paul, T., Pop, I. (2006). Natural convection in a square cavity filled with a porous medium: Effects of various thermal boundary conditions. International Journal of Heat and Mass Transfer, 49(7-8): 1430-1441.

http://dx.doi.org/10.1016/j.ijheatmasstransfer.2005.09.0 18

[5] Tong, T.W., Subramanian, E. (1985). A boundary-layer analysis for natural convection in vertical porous enclosures-use of the Brinkman-extended Darcy model. International Journal of Heat and Mass Transfer, 28(3): 563-571. https://doi.org/10.1016/0017-9310(85)90179-6

[6] Lauriat, G., Prasad, V. (1987). Natural convection in a vertical porous cavity: A numerical study for Brinkmanextended Darcy formulation. Journal of Heat Transfer, 109(3): 295-320. http://dx.doi.org/10.1115/1.3248143

[7] Brinkman, H.C. (1947). On the permeability of media consisting of closely packed porous interface. Appl. Sci. Res., 27-34.

[8] Mehdaoui, R., Elmir, M., Draoui, B., Imine, O., Mojtabi, A. (2008). Comparative study between the DarcyBrinkman model and the modified Navier-Stokes equations in the case of natural convection in a porous cavity. Leonardo Journal of Sciences, 1(12): 121-134.

[9] Choi, S.U., Eastman, J.A. (1995). Enhancing thermal conductivity of fluids with nanoparticles (No. ANL/MSD/CP-84938; CONF-951135-29). Argonne National Lab., IL (United States).

[10] Khanafer, K., Vafai, K., Lightstone, M. (2003).
Buoyancy-driven heat transfer enhancement in a twodimensional enclosure utilizing nanofluids. International Journal of Heat and Mass Transfer, 46(19): 3639-3653. http://dx.doi.org/10.1016/S0017-9310(03)00156-X

[11] Khanafer, K., Vafai, K. (2019). Applications of nanofluids in porous medium. Journal of Thermal Analysis and Calorimetry, 135(2): 1479-1492. https://doi.org/10.1007/s10973-018-7565-4

[12] Sheikhzadeh, G.A., Nazari, S. (2013). Numerical study of natural convection in a square cavity filled with a porous medium saturated with nanofluid. Transport Phenomena in Nano and Micro Scales, 1(2): 138-146. http://dx.doi.org/10.7508/TPNMS.2013.02.007

[13] Sheikholeslami, M. (2017). Numerical simulation of magnetic nanofluid natural convection in porous media. Physics Letters A, 381(5): 494-503. http://dx.doi.org/10.1016/j.physleta.2016.11.042

[14] Sheikholeslami, M., Shehzad, S.A. (2017). Magnetohydrodynamic nanofluid convection in a porous enclosure considering heat flux boundary condition. International Journal of Heat and Mass Transfer, 106: 1261-1269.

https://doi.org/10.1016/j.ijheatmasstransfer.2016.10.107

[15] Groşan, T., Revnic, C., Pop, I., Ingham, D.B. (2015). Free convection heat transfer in a square cavity filled with a porous medium saturated by a nanofluid. International Journal of Heat and Mass Transfer, 87: 3641.

http://dx.doi.org/10.1016/j.ijheatmasstransfer.2015.03.0 78

[16] Buongiorno, J. (2006). Convective transport in nanofluids. Journal of Heat Transfer, 128(3): 240-250. http://dx.doi.org/10.1115/1.2150834

[17] Baïri, A. (2019). Experimental study on enhancement of free convective heat transfer in a tilted hemispherical enclosure by using Water-ZnO nanofluid saturated porous materials. Applied Thermal Engineering, 148: 992-998. https://doi.org/10.1016/j.applthermaleng.2018.11.115

[18] Rahman, M.M., Pop, I., Saghir, M.Z. (2019). Steady free convection flow within a titled nanofluid saturated porous cavity in the presence of a sloping magnetic field energized by an exothermic chemical reaction administered by Arrhenius kinetics. International Journal of Heat and Mass Transfer, 129: 198-211. http://dx.doi.org/10.1016/j.ijheatmasstransfer.2018.09.1 05

[19] Rashad, A.M., Armaghani, T., Chamkha, A.J., Mansour, M.A. (2018). Entropy generation and MHD natural convection of a nanofluid in an inclined square porous cavity: Effects of a heat sink and source size and location. Chinese Journal of Physics, 56(1): 193-211. https://doi.org/10.1016/j.cjph.2017.11.026

[20] Chamkha, A.J., Rashad, A.M., Armaghani, T., Mansour, M.A. (2018). Effects of partial slip on entropy generation and MHD combined convection in a lid-driven porous enclosure saturated with a $\mathrm{Cu}$-water nanofluid. Journal of Thermal Analysis and Calorimetry, 132(2): 1291. https://doi.org/1306. 10.1007/s10973-017-6918-8

[21] Shenoy, A., Sheremet, M., Pop, I. (2016). Convective Flow and Heat Transfer from Wavy Surfaces: Viscous Fluids, Porous Media, and Nanofluids. CRC Press.

[22] Sheremet, M.A., Pop, I., Shenoy, A. (2015). Unsteady free convection in a porous open wavy cavity filled with 
a nanofluid using Buongiorno's mathematical model. International Communications in Heat and Mass Transfer, 67:

66-72. http://dx.doi.org/10.1016/j.icheatmasstransfer.2015.07.0 07

[23] Martyushev, S.G., Sheremet, M.A. (2014). Conjugate natural convection combined with surface thermal radiation in an air filled cavity with internal heat source. International Journal of Thermal Sciences, 76: 51-67. http://dx.doi.org/10.1016/j.ijthermalsci.2013.08.012

[24] Sheremet, M.A., Oztop, H.F., Pop, I., Al-Salem, K. (2016). MHD free convection in a wavy open porous tall cavity filled with nanofluids under an effect of corner heater. International Journal of Heat and Mass Transfer, 103:

955-964 http://dx.doi.org/10.1016/j.ijheatmasstransfer.2016.08.0 06

\section{NOMENCLATURE}

$H \quad$ Cavity width

$L \quad$ Cavity height

A Aspect Ratio, L/H

$w \quad$ Wavelength of wavy surface

lw Length of wavy surface

$h \quad$ Height of the source

a Corrugated source amplitude

$n \quad$ Wavelength number

$(x, y) \quad$ Cartesian coordinates

$(u, v) \quad$ Components of velocity fields

$T \quad$ Temperature

$P \quad$ Pressure

$g \quad$ Gravitational acceleration $k^{*} \quad$ Permeability

$k \quad$ Thermal conductivity

$R_{k} \quad$ Thermal conductivity ratio

$R_{\alpha} \quad$ Thermal diffusivity ratio

Da Darcy number

$\mathrm{Pr} \quad$ Prandlt number

$R a \quad$ Rayleigh number

$R a^{*} \quad$ Modified Rayleigh number

$\mathrm{Nu} \quad$ Nusselt number

\section{Greek symbols}

$\alpha \quad$ Thermal diffusivity

$\rho \quad$ Density

$\beta \quad$ Thermal expansion coefficient

$\varepsilon \quad$ Ratio of heating element to enclosure width, w/H

$\phi \quad$ Nanoparticles volume fraction

$\mu \quad$ Dynamic viscosity

$\psi \quad$ Stream function

( $\rho . C p)$ Heat capacity

\section{Subscripts}

$f \quad$ Fluid properties

nf Nanofluid properties

$s \quad$ Solid properties

c $\quad$ Cold wall

$h \quad$ Hot wall

$m \quad$ Porous medium

* Dimensionless parameters

avr Average

$L \quad$ Local 